\title{
Description of Deleya gen. nov. Created to Accommodate the Marine Species Alcaligenes aestus, A. pacificus, A. cupidus, A. venustus, and Pseudomonas marina
}

\author{
LINDA BAUMANN, RON D. BOWDITCH, AND PAUL BAUMANN* \\ Department of Bacteriology, University of California, Davis, California 95616
}

\begin{abstract}
Recent investigations of the evolutionary relationships of marine species assigned to the genera Alcaligenes (Alcaligenes aestus, Alcaligenes pacificus, Alcaligenes cupidus, and Alcaligenes venustus) and Pseudomonas (Pseudomonas marina) are reviewed. Data obtained by a number of different methods indicate that these five species are part of a single evolutionary lineage distinct from their close relatives, the fluorescent pseudomonads, and unrelated to the type species of Alcaligenes (Alcaligenes faecalis). Consequently, all five species are transferred to a new genus, Deleya, with Deleya aesta designated as the type species. The phenotype properties of $D$. aesta, Deleya pacifica, Deleya cupida, Deleya venusta, and Deleya marina are presented, together with the distinguishing traits of these organisms.
\end{abstract}

In 1972 we published a taxonomic study of gram-negative, nonfermentative marine bacteria which were motile by flagella and had rigid cell walls (4). Among the species described were four organisms with peritrichous flagella and guanine-plus-cytosine $(\mathrm{G}+\mathrm{C})$ contents in their deoxyribonucleic acids (DNAs) of 52 to 68 $\mathrm{mol} \%$. By a process of elimination, these organisms were assigned to the genus Alcaligenes, which, at that time, was defined primarily by peritrichous flagellation and obligatory respiratory metabolism (4). We recognized that this was a temporary expedient since the defining characters of the genus Alcaligenes were so few that further study of these species, as well as other species of Alcaligenes, would be required to determine the validity of the assignment.

In the last 5 years, studies of evolutionary relationship by analysis of the highly conserved sequences of ribosomal ribonucleic acid (rRNA) have created major problems with respect to the utility and function of generic assignments. Ideally, a genus should reflect the evolutionary relationships of the constituent species and be characterized by simple, readily determinable traits. In practice, these two goals may not be readily attainable, as is well illustrated in the taxonomy of the genera Pseudomonas and Alcaligenes.

Palleroni et al. (16) were the first to systematically apply in vitro rRNA-DNA hybridization to studies of Pseudomonas taxonomy. The results of these authors delineated five major rRNA homology groups, some of which were more distantly related to each other than to Escherichia coli. The possibility of splitting Pseudomonas into five different genera was considered but rejected since it was not possible to distinguish the rRNA homology groups by simple, readily determinable, universally positive or negative traits $(16,20)$. Furthermore, the major evolutionary lineages in procaryotes had not yet been established, so that practical considerations (such as ease of generic assignment of species) overruled the more attractive goal of a natural classification. As more information on procaryotic lineages has become available, primarily from the laboratories of C. R. Woese and J. De Ley and their collaborators, the practical solution has become less tenable $(10-15,19)$ since it is clearly more desirable to have genera that reflect evolutionary relationships. The latter alternative has recently been chosen by Swings et al. (22), who transferred Pseudomonas maltophilia into the genus Xanthomonas, thereby joining two members of rRNA homology group $\mathrm{V}$ of Palleroni et al. (16). The consequence of this transfer is that Xanthomonas and Pseudomonas cannot be distinguished by simple phenotypic traits. Instead, the new definition of Xanthomonas is based on the sequence similarities of rRNAs as reflected in heteroduplex stability. Hopefully, future research will provide less difficult means of identifying such genera, such as a simple method for detecting specific, characteristic sequences of informational molecules.

A situation similar to that in Pseudomonas exists with Alcaligenes, which has proven to be heterogeneous. Many of the species assigned to this genus by Davis et al. (9) resembled Pseudomonas in their general properties but differed by 
TABLE 1. Phenotypic properties not universally present in Deleya species $^{a}$

\begin{tabular}{|c|c|c|c|c|c|c|}
\hline \multirow[b]{2}{*}{ Trait } & \multicolumn{3}{|c|}{ D. aesta } & \multicolumn{3}{|c|}{ D. cupida } \\
\hline & $\begin{array}{l}\text { Phenotype } \\
\text { of species } \\
(n=6)^{b}\end{array}$ & $\begin{array}{c}\text { Phenotype of } \\
\text { strain } 134^{\mathrm{T}} \\
\left(=\text { ATCC } 27128^{\mathrm{T}}\right)\end{array}$ & $\begin{array}{l}\text { Strain(s) which } \\
\text { is positive or } \\
\text { negative }\end{array}$ & $\begin{array}{l}\text { Phenotype } \\
\text { of species } \\
(n=5)\end{array}$ & $\begin{array}{l}\text { Phenotype of } \\
\text { strain } 79^{\mathrm{T}} \\
\text { (= ATCC } 27124^{\mathrm{T}} \text { ) }\end{array}$ & $\begin{array}{l}\text { Strain(s) which } \\
\text { is positive or } \\
\text { negative }\end{array}$ \\
\hline Flagellation ${ }^{c}$ & $\operatorname{Pr}$ & $\operatorname{Pr}$ & & $\operatorname{Pr}$ & $\operatorname{Pr}$ & \\
\hline Motility & $+^{d}$ & + & & $+^{d}$ & + & \\
\hline Oxidase & + & + & & - & - & \\
\hline $\mathrm{NO}_{3}{ }^{-} \rightarrow \mathrm{NO}_{2}^{-}$ & (5) & + & $(-) 138^{e}$ & (4) & + & $(-) 83^{e}$ \\
\hline \multicolumn{7}{|l|}{ Growth at: } \\
\hline $4^{\circ} \mathrm{C}$ & - & - & & - & - & \\
\hline $40^{\circ} \mathrm{C}$ & + & + & & 3 & - & $(-) 79,80$ \\
\hline $45^{\circ} \mathrm{C}$ & 1 & + & $(+) 134$ & - & - & \\
\hline \multicolumn{7}{|l|}{ Production of: } \\
\hline Amylase & 2 & + & $(+) 133,134$ & - & - & \\
\hline Alginase & - & - & & - & - & \\
\hline \multicolumn{7}{|l|}{ Utilization of: } \\
\hline D-Ribose & - & - & & (4) & + & $(-) 83$ \\
\hline D-Xylose & - & - & & (4) & + & $(-) 83$ \\
\hline D-Arabinose & - & - & & 2 & - & $(+) 80,81$ \\
\hline L-Arabinose & - & - & & + & + & \\
\hline L-Rhamnose & - & - & & 2 & - & $(+) 80,83$ \\
\hline D-Mannose & - & - & & + & + & \\
\hline D-Galactose & 3 & + & $\begin{array}{l}(+) 134,135 \\
138\end{array}$ & + & + & \\
\hline D-Fructose & + & + & & + & + & \\
\hline Sucrose & 3 & - & $\begin{array}{c}(+) 133,136 \\
137\end{array}$ & 3 & + & $(-) 81,82$ \\
\hline Trehalose & 3 & - & $\begin{array}{l}(+) 133,136 \\
137\end{array}$ & 3 & + & $(-) 81,82$ \\
\hline Maltose & 3 & - & $\begin{array}{l}(+) 133,136 \\
137\end{array}$ & 3 & + & $(-) 81,82$ \\
\hline Cellobiose & - & - & & 3 & + & $(-) 81,82$ \\
\hline Mellibiose & - & - & & 2 & + & $(+) 79,80$ \\
\hline Lactose & - & - & & 2 & + & $(+) 79,80$ \\
\hline Salicin & - & - & & 3 & + & (-) 81,82 \\
\hline Gluconate & (5) & + & $(-) 133$ & + & + & \\
\hline Saccharate & - & - & & + & + & \\
\hline Glucuronate & - & - & & + & + & \\
\hline Galacturonate & - & - & & + & + & \\
\hline$N$-Acetylglucosamine & - & - & & + & + & \\
\hline Butyrate & + & + & & + & + & \\
\hline Isobutyrate & + & + & & + & + & \\
\hline Valerate & + & + & & + & + & \\
\hline Isovalerate & + & + & & + & + & \\
\hline Caproate & + & + & & + & + & \\
\hline Heptanoate & + & + & & + & + & \\
\hline Caprylate & + & + & & + & + & \\
\hline Pelargonate & 1 & - & $(+) 137$ & + & + & \\
\hline Caprate & 3 & - & $\begin{array}{c}(+) 133,135 \\
136\end{array}$ & + & + & \\
\hline Malonate & 1 & - & $(+) 135$ & 3 & + & $(-) 81,83$ \\
\hline
\end{tabular}

their peritrichous flagellation. A close relationship between several of these species and Pseudomonas rRNA homology groups II and III has since been established by studies of rRNA sequence similarity $(10-15,19,22)$, regulation of key enzymes of aromatic amino acid biosynthesis $(7,23)$, and evolution of glutamine synthetase (3). The type species of Alcaligenes (Alcaligenes faecalis) has been found to belong to a separate lineage closely related to the genus Bordetella $(11,13,22)$. It is probable that rRNA homology groups II and III, along with their respective Alcaligenes species, will eventually become two new genera containing both polarly and peritrichously flagellated organisms.

The rRNA homology studies of De Ley and 
TABLE 1-Continued

\begin{tabular}{|c|c|c|c|c|c|c|c|c|}
\hline \multicolumn{3}{|c|}{ D. pacifica } & \multicolumn{3}{|c|}{ D. venusta } & \multicolumn{3}{|c|}{ D. marina } \\
\hline $\begin{array}{l}\text { Pheno- } \\
\text { type of } \\
\text { species } \\
(n=6)\end{array}$ & $\begin{array}{c}\text { Phenotype } \\
\text { of strain } 62^{\mathrm{T}} \\
(=\mathrm{ATCC} \\
\left.27122^{\mathrm{T}}\right)\end{array}$ & $\begin{array}{l}\text { Strain(s) which } \\
\text { is positive or } \\
\text { negative }\end{array}$ & $\begin{array}{l}\text { Pheno- } \\
\text { type of } \\
\text { species } \\
(n=14)\end{array}$ & $\begin{array}{c}\text { Phenotype } \\
\text { of strain } 86^{\mathrm{T}} \\
(=\mathrm{ATCC} \\
\left.27125^{\mathrm{T}}\right)\end{array}$ & $\begin{array}{l}\text { Strain(s) which } \\
\text { is positive or } \\
\text { negative }\end{array}$ & $\begin{array}{l}\text { Pheno- } \\
\text { type of } \\
\text { species } \\
(n=7)\end{array}$ & $\begin{array}{c}\text { Phenotype } \\
\text { of strain } 219^{\mathrm{T}} \\
(=\text { ATCC } \\
\left.25374^{\mathrm{T}}\right)\end{array}$ & $\begin{array}{l}\text { Strain(s) which } \\
\text { is positive or } \\
\text { negative }\end{array}$ \\
\hline Pr & $\operatorname{Pr}$ & & $\operatorname{Pr}$ & $\operatorname{Pr}$ & & $\mathbf{P}$ & & \\
\hline$+^{d}$ & + & & $+^{d}$ & + & & $5^{d}$ & - & $(-) 141,219^{e}$ \\
\hline+ & + & & + & + & & - & - & \\
\hline- & - & & + & + & & - & - & \\
\hline- & - & & + & + & & (6) & + & $(-) 140$ \\
\hline+ & + & & + & + & & 1 & + & $(+) 219$ \\
\hline (5) & + & $(-) 60^{e}$ & - & - & & - & - & \\
\hline- & - & & - & - & & - & - & \\
\hline- & - & & - & - & & 1 & - & $(+) 143$ \\
\hline - & - & & - & - & & + & + & \\
\hline 1 & - & $(+) 65$ & - & - & & - & - & \\
\hline- & - & & - & - & & - & - & \\
\hline- & - & & - & - & & - & - & \\
\hline - & - & & - & - & & - & - & \\
\hline- & - & & - & - & & - & - & \\
\hline- & - & & - & - & & + & + & \\
\hline+ & + & & (11) & + & $(-) 88^{e}$ & + & + & \\
\hline- & - & & 9 & + & $\begin{array}{r}\text { (-) } 88,91, \\
92,95,97\end{array}$ & - & - & \\
\hline- & - & & 9 & + & $\begin{array}{l}(-) 88,91 \\
92,95,97\end{array}$ & - & - & \\
\hline- & - & & 9 & + & $\begin{array}{l}\text { (-) } 88,91, \\
92,95,97\end{array}$ & - & - & \\
\hline- & - & & - & - & & - & - & \\
\hline- & - & & - & - & & - & - & \\
\hline- & - & & - & - & & - & - & \\
\hline- & - & & - & - & & - & - & \\
\hline+ & + & & (13) & + & $(-) 89$ & + & + & \\
\hline- & - & & - & - & & - & - & \\
\hline- & - & & 4 & + & $(+) 85-88$ & - & - & \\
\hline- & - & & 5 & + & $(+) 84-88$ & - & - & \\
\hline+ & + & & 10 & + & $\begin{array}{c}(-) 85,93 \\
96,97\end{array}$ & - & - & \\
\hline+ & + & & + & + & & (6) & + & $(-) 139$ \\
\hline+ & + & & + & + & & 1 & - & $(+) 141$ \\
\hline (5) & + & $(-) 61$ & + & + & & (6) & + & (+) 139 \\
\hline+ & + & & + & + & & (6) & + & (+) 139 \\
\hline+ & + & & + & + & & 5 & + & (-) 139,140 \\
\hline+ & + & & 9 & + & (-) 93-97 & + & + & \\
\hline 4 & - & $(-) 62,65$ & (13) & + & $(-) 97$ & 6 & + & $(-) 142$ \\
\hline (5) & - & $(-) 62$ & (13) & - & $(-) 86$ & + & + & \\
\hline 4 & - & $(-) 61,62$ & + & + & & + & + & \\
\hline - & - & & 4 & - & $\begin{array}{c}(+) 84,85 \\
87,97\end{array}$ & 5 & + & (-) 139,140 \\
\hline
\end{tabular}

Continued on next page

collaborators $(10,11,15,22)$ have revealed further heterogeneity within Alcaligenes by showing that the marine Alcaligenes species, which are the subject of this paper, constitute a sublineage in an evolutionary branch that gave rise to Pseudomonas rRNA homology group I, which contains such well-studied species as Pseudomonas aeruginosa, Pseudomonas pu- tida, Pseudomonas fluorescens, and Pseudomonas stutzeri (16). Our own findings, based on patterns of regulation of aspartokinase activities, are in agreement with a relationship between the marine species of Alcaligenes and the species in Pseudomonas rRNA homology group I $(2,8)$. Studies of carbohydrate catabolism have shown that marine species of Alcaligenes are 
TABLE 1-Continued

\begin{tabular}{|c|c|c|c|c|c|c|}
\hline \multirow[b]{2}{*}{ Trait } & \multicolumn{3}{|c|}{ D. aesta } & \multicolumn{3}{|c|}{ D. cupida } \\
\hline & $\begin{array}{l}\text { Phenotype } \\
\text { of species } \\
(n=6)^{b}\end{array}$ & $\begin{array}{c}\text { Phenotype of } \\
\text { strain } 134^{\mathrm{T}} \\
\left(=\text { ATCC } 27128^{\mathrm{T}}\right)\end{array}$ & $\begin{array}{l}\text { Strain(s) which } \\
\text { is positive or } \\
\text { negative }\end{array}$ & $\begin{array}{c}\text { Phenotype } \\
\text { of species } \\
(n=5)\end{array}$ & $\begin{array}{c}\text { Phenotype of } \\
\text { strain } 79^{\mathrm{T}} \\
\left(=\text { ATCC } 27124^{\mathrm{T}}\right)\end{array}$ & $\begin{array}{l}\text { Strain(s) which } \\
\text { is positive or } \\
\text { negative }\end{array}$ \\
\hline Glutarate & 4 & - & (-) 133,134 & 2 & - & (+) 82,83 \\
\hline Adipate & (5) & + & $(-) 133$ & - & - & \\
\hline Pimelate & + & + & & - & - & \\
\hline Suberate & + & + & & - & - & \\
\hline Azelate & + & + & & - & - & \\
\hline Sebacate & + & + & & - & - & \\
\hline DL-Malate & + & + & & + & + & \\
\hline D-(-)-Tartrate & - & - & & 1 & - & $(+) 80$ \\
\hline $\mathrm{L}-(+)$-Tartrate & 4 & + & (-) 135,136 & 3 & - & (-) 79,83 \\
\hline meso-Tartrate & 2 & - & $(+) 137,138$ & (4) & + & $(-) 83$ \\
\hline Glycolate & - & - & & + & + & \\
\hline DL-Glycerate & + & + & & + & + & \\
\hline Citrate & 3 & - & $\begin{array}{l}(+) 135,136, \\
138\end{array}$ & + & + & \\
\hline$\alpha$-Ketoglutarate & 4 & - & $(-) 133,134$ & + & + & \\
\hline Aconitate & - & - & & + & + & \\
\hline Laevulinate & 3 & + & $(+)$ 133-135 & - & - & \\
\hline Citraconate & 1 & - & $(+) 135$ & - & - & \\
\hline Itaconate & 1 & - & $(+) 138$ & - & - & \\
\hline Mesaconate & 1 & - & $(+) 138$ & - & - & \\
\hline Erythritol & 4 & + & $(-) 135,137$ & 2 & + & $(+) 79,80$ \\
\hline Mannitol & + & + & & + & + & \\
\hline Sorbitol & + & + & & (4) & + & $(-) 82$ \\
\hline meso-Inositol & 2 & + & (+) 133,134 & 3 & + & $(-) 81,83$ \\
\hline Adonitol & - & - & & 3 & + & $(-) 82,83$ \\
\hline Ethyleneglycol & - & - & & 2 & - & $(+) 80,82$ \\
\hline Propyleneglycol & - & - & & - & - & \\
\hline 2,3 -Butyleneglycol & 3 & - & $\begin{array}{c}(+) 133,137, \\
138\end{array}$ & - & - & \\
\hline Ethanol & + & + & & (4) & + & $(-) 83$ \\
\hline n-Propanol & + & + & & (4) & + & $(-) 83$ \\
\hline$n$-Butanol & + & + & & 3 & - & $(-) 79,83$ \\
\hline Isobutanol & (5) & + & $(-) 135$ & (4) & + & $(-) 83$ \\
\hline L-Mandelate & - & - & & 1 & - & $(+) 83$ \\
\hline Benzoylformate & - & - & & 2 & + & $(+) 79,83$ \\
\hline Benzoate & - & - & & + & + & \\
\hline$o \cdot$ Hydroxybenzoate & - & - & & 1 & - & $(+) 83$ \\
\hline$m$-Hydroxybenzoate & - & - & & 1 & - & $(+) 83$ \\
\hline$p$-Hydroxybenzoate & - & - & & + & + & \\
\hline
\end{tabular}

distinctive in that they utilize D-fructose via a Dfructose:phosphotransferase system and 1-phosphofructokinase, followed by the conversion of the resulting fructose 1,6-diphosphate to fructose 6-phosphate by means of a pyrophosphatedependent 6-phosphofructokinase, with subsequent catabolism proceeding via the EntnerDoudoroff pathway (17). This combination of enzyme activities, together with a lack of fruc- tose-1,6-diphosphate phosphatase, has been found in Pseudomonas marina but in no other marine or terrestrial Pseudomonas or Alteromonas species tested $(5,17,18)$. The close relationship between $P$. marina, a polarly flagellated species, and the marine Alcaligenes species, as well as the more distant relationship of these organisms to Pseudomonas rRNA homology group I, has been confirmed by evolutionary 
TABLE 1-Continued

\begin{tabular}{|c|c|c|c|c|c|c|c|c|}
\hline \multicolumn{3}{|c|}{ D. pacifica } & \multicolumn{3}{|c|}{ D. venusta } & \multicolumn{3}{|c|}{ D. marina } \\
\hline $\begin{array}{l}\text { Pheno- } \\
\text { type of } \\
\text { species } \\
(n=6)\end{array}$ & $\begin{array}{c}\text { Phenotype } \\
\text { of strain } 62^{\mathrm{T}} \\
(=\mathrm{ATCC} \\
\left.27122^{\mathrm{T}}\right)\end{array}$ & $\begin{array}{l}\text { Strain(s) which } \\
\text { is positive or } \\
\text { negative }\end{array}$ & $\begin{array}{l}\text { Pheno- } \\
\text { type of } \\
\text { species } \\
(n=14)\end{array}$ & $\begin{array}{c}\text { Phenotype } \\
\text { of strain } 86^{\mathrm{T}} \\
(=\mathrm{ATCC} \\
\left.27125^{\mathrm{T}}\right)\end{array}$ & $\begin{array}{l}\text { Strain(s) which } \\
\text { is positive or } \\
\text { negative }\end{array}$ & $\begin{array}{l}\text { Pheno- } \\
\text { type of } \\
\text { species } \\
(n=7)\end{array}$ & $\begin{array}{l}\text { Phenotype } \\
\text { of strain } 219^{\mathrm{T}} \\
(=\text { ATCC } \\
\left.25374^{\mathrm{T}}\right)\end{array}$ & $\begin{array}{l}\text { Strain(s) which } \\
\text { is positive or } \\
\text { negative }\end{array}$ \\
\hline+ & + & & + & + & & - & - & \\
\hline- & - & & 7 & - & $\begin{array}{l}(+) 84,85 \\
\quad 89,90,93- \\
95\end{array}$ & - & - & \\
\hline- & - & & 1 & - & $(+) 85$ & - & - & \\
\hline- & - & & - & - & & - & - & \\
\hline- & - & & 3 & - & $\begin{array}{c}(+) 93,94, \\
95\end{array}$ & - & - & \\
\hline- & - & & 1 & - & $(+) 85$ & - & - & \\
\hline (5) & - & $(-) 62$ & + & + & & + & + & \\
\hline - & - & & - & - & & - & - & \\
\hline- & - & & - & - & & 2 & - & (+) 141,142 \\
\hline - & - & & - & - & & - & - & \\
\hline - & - & & - & - & & - & - & \\
\hline (5) & + & $(-) 65$ & 9 & - & $(-)$ 84-88 & + & + & \\
\hline+ & + & & + & + & & + & + & \\
\hline+ & + & & + & + & & + & + & \\
\hline (5) & + & $(-) 61$ & (13) & + & $(-) 88$ & + & + & \\
\hline 1 & - & $(+) 63$ & - & - & & - & - & \\
\hline - & - & & - & - & & - & - & \\
\hline - & - & & 1 & - & (+) 91 & - & - & \\
\hline - & - & & - & - & & - & - & \\
\hline - & - & & 1 & - & (+) 88 & - & - & \\
\hline - & - & & (12) & + & (-) 88,97 & + & + & \\
\hline - & - & & (12) & + & $(-) 88,97$ & - & - & \\
\hline - & - & & + & + & & 5 & + & (-) 139,141 \\
\hline 1 & - & (+) 65 & - & - & & - & - & \\
\hline - & - & & - & - & & - & - & \\
\hline - & - & & 5 & + & (+) $84-88$ & - & - & \\
\hline 1 & + & $(+) 62$ & 8 & + & $(-) 92-97$ & 3 & - & $\begin{array}{l}\text { (+) } 141,143 \text {, } \\
144\end{array}$ \\
\hline - & - & & + & + & & 5 & + & (-) 141,142 \\
\hline - & - & & + & + & & 5 & - & (-) 141,142 \\
\hline- & - & & + & + & & 1 & + & (+) 219 \\
\hline- & - & & + & + & & - & - & \\
\hline- & - & & 4 & + & $\begin{array}{c}(+) 86-88, \\
92\end{array}$ & - & - & \\
\hline- & - & & 4 & + & $\begin{array}{c}(+) 86-88, \\
92\end{array}$ & - & - & \\
\hline+ & + & & 5 & + & $\begin{array}{c}(+) 86-88, \\
92,97\end{array}$ & - & - & \\
\hline- & - & & 4 & + & $\begin{array}{c}(+) 86-88, \\
92\end{array}$ & - & - & \\
\hline 2 & - & $(+) 60,61$ & 1 & + & $(+) 86$ & - & - & \\
\hline 3 & + & $(+) 60-62$ & 5 & + & $\begin{array}{c}(+) 86-88 \\
92,95\end{array}$ & - & - & \\
\hline
\end{tabular}

Continued on next page

studies of glutamine synthetase and iron-containing superoxide dismutase (E. F. DeLong, L. Baumann, R. D. Bowditch, and P. Baumann, Arch. Microbiol., in press). Since the work of J. De Ley has provided the primary evidence of a distinct lineage for the marine species of Alcaligenes $(10,11,15,22)$, we propose to place all of these species, as well as $P$. marina, into a new genus designated Deleya.

\section{MATERIALS AND METHODS}

We have previously described the methods of isolation and the sources of the strains used in this study, as 
TABLE 1-Continued

\begin{tabular}{|c|c|c|c|c|c|c|}
\hline \multirow[b]{2}{*}{ Trait } & \multicolumn{3}{|c|}{ D. aesta } & \multicolumn{3}{|c|}{ D. cupida } \\
\hline & $\begin{array}{l}\text { Phenotype } \\
\text { of species } \\
(n=6)^{b}\end{array}$ & $\begin{array}{c}\text { Phenotype of } \\
\text { strain } 134^{\mathrm{T}} \\
\left.\text { (= ATCC } 27128^{\mathrm{T}}\right)\end{array}$ & $\begin{array}{l}\text { Strain(s) which } \\
\text { is positive or } \\
\text { negative }\end{array}$ & $\begin{array}{l}\text { Phenotype } \\
\text { of species } \\
(n=5)\end{array}$ & $\begin{array}{c}\text { Phenotype of } \\
\text { strain } 79^{\mathrm{T}} \\
\left(=\text { ATCC } 27124^{T}\right)\end{array}$ & $\begin{array}{l}\text { Strain(s) which } \\
\text { is positive or } \\
\text { negative }\end{array}$ \\
\hline Phenylacetate & 2 & - & (+) 135,138 & - & - & \\
\hline Quinate & - & - & & 2 & - & $(+) 80,81$ \\
\hline Glycine & - & - & & 3 & - & $(-) 79,81$ \\
\hline$\beta$-Alanine & 1 & - & $(+) 138$ & (4) & + & $(-) 83$ \\
\hline L-Serine & - & - & & + & + & \\
\hline L-Threonine & - & - & & 1 & - & $(+) 83$ \\
\hline L-Leucine & 3 & + & $\begin{array}{c}(+) 134,136 \text {, } \\
137\end{array}$ & (4) & + & $(-) 83$ \\
\hline L-Isoleucine & + & + & & 2 & - & $(+) 80,81$ \\
\hline L-Valine & 2 & - & $(+) 136,138$ & - & - & \\
\hline L-Aspartate & (5) & - & $(-) 134$ & + & + & \\
\hline L-Lysine & + & + & & + & + & \\
\hline L-Arginine & + & + & & + & + & \\
\hline L-Ornithine & 4 & - & $(-) 133,134$ & 3 & + & $(-) 81,83$ \\
\hline$\delta$-Aminovalerate & - & - & & (4) & - & $(-) 79$ \\
\hline L-Histidine & - & - & & (4) & + & $(-) 81$ \\
\hline L-Tyrosine & - & - & & + & + & \\
\hline L-Phenylalanine & - & - & & 2 & - & (+) 82,83 \\
\hline L-Tryptophan & - & - & & - & - & \\
\hline DL-Kynurenine & - & - & & - & - & \\
\hline Kynurenate & - & - & & 2 & - & $(+) 80,83$ \\
\hline Anthranilate & - & - & & 1 & - & $(+) 83$ \\
\hline Ethanolamine & - & - & & - & - & \\
\hline Benzylamine & - & - & & - & - & \\
\hline Putrescine & - & - & & + & + & \\
\hline Spermine & - & - & & 2 & + & $(+) 79,82$ \\
\hline Histamine & - & - & & 3 & - & (-) 79,82 \\
\hline Butylamine & - & - & & - & - & \\
\hline Pentylamine & - & - & & 2 & - & (+) 81,82 \\
\hline Betaine & - & - & & + & + & \\
\hline Sarcosine & - & - & & + & + & \\
\hline Creatine & 1 & - & $(+) 135$ & 1 & - & $(+) 82$ \\
\hline Hippurate & - & - & & (4) & + & $(-) 82$ \\
\hline Acetamide & - & - & & - & - & \\
\hline Nicotinate & - & - & & - & - & \\
\hline Nicotinamide & - & - & & - & - & \\
\hline Trigonelline & - & - & & - & - & \\
\hline Allantoin & - & - & & + & + & \\
\hline Adenine & - & - & & - & - & \\
\hline Cytosine & 1 & + & $(+) 134$ & - & - & \\
\hline Uracil & 1 & + & $(+) 134$ & - & - & \\
\hline
\end{tabular}

${ }^{a}$ The following strains were studied: $D$. aesta strains 133 through $138 ; D$. cupida strains 79 through $83 ; D$. pacifica strains 60 through $65 ; D$. venusta strains 84 through 97; and D. marina strains 139 through 144 and 219.

${ }^{b}$ Number of strains studied.

${ }^{c}$ Pr, Peritrichous; P, polar.

${ }^{d}+$, All strains positive; - all strains negative. The numbers indicate the numbers of positive strains; parentheses indicate that $80 \%$ or more of the strains are positive.

$e(-)$, Strain(s) indicated is negative; $(+)$, strain(s) indicated is positive. 
TABLE 1-Continued

\begin{tabular}{|c|c|c|c|c|c|c|c|c|}
\hline \multicolumn{3}{|c|}{ D. pacifica } & \multicolumn{3}{|c|}{ D. venusta } & \multicolumn{3}{|c|}{ D. marina } \\
\hline $\begin{array}{l}\text { Pheno- } \\
\text { type of } \\
\text { species } \\
(n=6)\end{array}$ & $\begin{array}{c}\text { Phenotype } \\
\text { of strain } 62^{\mathrm{T}} \\
(=\text { ATCC } \\
\left.27122^{\mathrm{T}}\right)\end{array}$ & $\begin{array}{l}\text { Strain(s) which } \\
\text { is positive or } \\
\text { negative }\end{array}$ & $\begin{array}{l}\text { Pheno- } \\
\text { type of } \\
\text { species } \\
(n=14)\end{array}$ & $\begin{array}{c}\text { Phenotype } \\
\text { of strain } 86^{\mathrm{T}} \\
(=\text { ATCC } \\
\left.27125^{\mathrm{T}}\right)\end{array}$ & $\begin{array}{l}\text { Strain(s) which } \\
\text { is positjve or } \\
\text { negative }\end{array}$ & $\begin{array}{l}\text { Pheno- } \\
\text { type of } \\
\text { species } \\
(n=7)\end{array}$ & $\begin{array}{c}\text { Phenotype } \\
\text { of strain } 219^{\mathrm{T}} \\
(=\text { ATCC } \\
\left.25374^{\mathrm{T}}\right)\end{array}$ & $\begin{array}{c}\text { Strain(s) which } \\
\text { is positive or } \\
\text { negative }\end{array}$ \\
\hline+ & + & & + & + & & - & - & \\
\hline- & - & & 4 & + & $\begin{array}{l}(+) 86-88 \\
92\end{array}$ & - & - & \\
\hline+ & + & & - & - & & - & - & \\
\hline 4 & - & $(-) 61,62$ & 8 & + & $(-)$ 89-94 & + & + & \\
\hline+ & + & & 2 & - & (+) 96,97 & + & + & \\
\hline- & - & & - & - & & - & - & \\
\hline$(5)$ & + & $(-) 63$ & (13) & + & $(-) 97$ & (6) & + & $(-) 139$ \\
\hline- & - & & 7 & + & $\begin{array}{c}(+) 84-88 \\
91,96\end{array}$ & 4 & - & $\begin{array}{l}(-) 139,140 \\
219\end{array}$ \\
\hline- & - & & (11) & + & $(-) 95-97$ & - & - & \\
\hline+ & + & & + & + & & + & + & \\
\hline+ & + & & + & + & & 2 & - & $(+) 141,142$ \\
\hline+ & + & & (12) & + & (-) 95,97 & 5 & + & $(-) 139,140$ \\
\hline+ & + & & 9 & + & $\begin{array}{l}(-) 89,92- \\
95\end{array}$ & 5 & + & $(-) 139,140$ \\
\hline+ & + & & + & + & & - & - & \\
\hline+ & + & & (12) & + & (-) 96,97 & - & - & \\
\hline+ & + & & + & + & & + & + & \\
\hline+ & + & & (13) & + & $(-) 97$ & 1 & + & (+) 219 \\
\hline- & - & & 6 & + & $\begin{array}{l}(+) 84,86 \\
87,89,90 \\
\quad 92\end{array}$ & - & - & \\
\hline+ & + & & - & - & & - & - & \\
\hline+ & + & & - & - & & - & - & \\
\hline+ & + & & 10 & + & $\begin{array}{c}(-) 84,85 \\
93,95\end{array}$ & - & - & \\
\hline- & - & & (13) & + & $(-) 97$ & - & - & \\
\hline (5) & + & $(-) 65$ & - & - & & - & - & \\
\hline+ & + & & + & + & & - & - & \\
\hline+ & + & & - & - & & - & - & \\
\hline+ & + & & 1 & - & $(+) 97$ & - & - & \\
\hline+ & + & & 8 & - & $\begin{array}{l}(-) 84-86, \\
88,93,94\end{array}$ & - & - & \\
\hline 1 & - & $(+) 65$ & - & - & & - & - & \\
\hline+ & + & & + & + & & 5 & + & (-) 139,142 \\
\hline+ & + & & + & + & & (6) & + & $(-) 140$ \\
\hline- & - & & - & - & & - & - & \\
\hline 4 & + & $(-) 60,61$ & 5 & + & $\begin{array}{c}(+) 86-88 \\
92,97\end{array}$ & - & - & \\
\hline- & - & & 3 & + & $\begin{array}{c}(+) 86,87 \\
96\end{array}$ & - & - & \\
\hline 2 & + & $(+) 61,62$ & + & + & & - & - & \\
\hline- & - & & 6 & - & $\begin{array}{l}\text { (+) } 84,95, \\
92-94,96\end{array}$ & - & - & \\
\hline 1 & - & $(+) 63$ & - & - & & - & - & \\
\hline (5) & - & $(-) 62$ & + & + & & - & - & \\
\hline 3 & - & $(+) 63-65$ & - & - & & - & - & \\
\hline- & - & & - & - & & - & - & \\
\hline- & - & & - & - & & - & - & \\
\hline
\end{tabular}

well as the details of the methodology used for phenotypic characterization $(4,6)$.

\section{RESULTS}

The following phenotypic properties were present in all strains: straight rods; accumulation of poly- $\beta$-hydroxybutyrate as an intracellular reserve product; growth at 20 to $35^{\circ} \mathrm{C}$; and utilization of $\mathrm{D}$-glucose, acetate, propionate, succinate, fumarate, $D L-\beta$-hydroxybutyrate, $D L$ lactate, pyruvate, glycerol, $L-\alpha$-alanine, $D-\alpha$ alanine, L-glutamate, and L-proline. 
TABLE 2. Some distinguishing properties of the species of Deleya

\begin{tabular}{lccccccccccc}
\hline \multicolumn{1}{c}{ Species } & $\begin{array}{c}\text { No. of } \\
\text { strains }\end{array}$ & Flagel- & $\begin{array}{l}\text { Oxi- } \\
\text { lation }^{a}\end{array}$ & dase & $\begin{array}{c}\text { Growth } \\
\text { at } 4^{\circ} \mathrm{C}\end{array}$ & $\begin{array}{l}\text { D-Ri- } \\
\text { bose }\end{array}$ & $\begin{array}{c}\text { L-Arabi- } \\
\text { nose }\end{array}$ & $\begin{array}{c}\text { D-Man- } \\
\text { nose }\end{array}$ & $\begin{array}{c}\text { Saccha- } \\
\text { rate }\end{array}$ & $\begin{array}{c}\text { Suber- } \\
\text { ate }\end{array}$ & $\begin{array}{c}\text { Glyco- } \\
\text { late }\end{array}$ \\
\hline D. aesta & 6 & $\mathrm{Pr}$ & $+b$ & - & - & - & - & - & + & - \\
D. cupida & 5 & $\mathrm{Pr}$ & - & - & $(4)$ & + & + & + & - & + \\
D. pacifica & 6 & $\mathrm{Pr}$ & + & - & - & - & - & - & - & - \\
D. venusta & 14 & $\mathrm{Pr}$ & + & + & - & - & - & - & - & - \\
D. marina & 7 & $\mathrm{P}$ & - & $(6)$ & + & - & - & - & - & - \\
\hline
\end{tabular}

${ }^{a} \mathrm{Pr}$, Pertrichous; $\mathrm{P}$, polar.

$b+$, All strains positive; - , all strains negative. The numbers in parentheses indicate the numbers of positive strains which represent over $80 \%$ of the strains.

The following phenotypic properties were absent from all strains: denitrification; ability to fix molecular nitrogen; growth with $\mathrm{CO}_{2}$ as the carbon source and $\mathrm{H}_{2}$ as the energy source; arginine dihydrolase; production of extracellular lipase, gelatinase, and chitinase; utilization of D-fucose, cellulose, inulin, mucate, formate, oxalate, maleate, methanol, isopropanol, Dmandelate, phenylethanediol, phenol, naphthaline, L-norleucine, L-citrulline, D-tryptophan, DL- $\gamma$-aminobutyrate, DL- $\delta$-aminovalerate, $m$ aminobenzoate, $p$-aminobenzoate, methylamine, tryptamine, 2-amylamine, pantothenate, guanine, thymine, and $n$-hexadecane.

The phenotypic properties which were not universally present or absent in the strains are presented in Table 1. The traits which distinguish species of Deleya are presented in Table 2.

\section{DISCUSSION}

Below are descriptions of the genus Deleya (De.ley'a. M. L. fem. n. Deleya named for Jozef De Ley, a Belgian microbiologist) and the species which constitute this genus.

Description of Deleya gen. nov. Straight rods 0.8 to $1.1 \mu \mathrm{m}$ wide and 1.5 to $3.0 \mu \mathrm{m}$ long. Accumulate poly- $\beta$-hydroxybutyrate as an intracellular reserve product. Involution forms are usually present in old cultures or under adverse conditions of cultivation. Do not form endospores or microcysts. Most species are motile by 4 to 12 peritrichous flagella; one species is motile by 2 to 5 polar flagella. Strict aerobes; molecular oxygen is a universal electron acceptor. Do not denitrify or fix molecular nitrogen. All species are chemoorganotrophs that are able to grow on a mineral medium containing a seawater base, $\mathrm{D}$ glucose, and $\mathrm{NH}_{4} \mathrm{Cl}$. $\mathrm{Na}^{+}$is an absolute requirement for all species; the minimal concentration necessary for optimal growth ranges from 75 to $200 \mathrm{mM}$. All species utilize D-glucose, acetate, succinate, DL- $\beta$-hydroxybutyrate, lactate, glycerol, and $\mathrm{L}-\alpha$-alanine. All species grow at $35^{\circ} \mathrm{C}$. No species has an extracellular lipase, gelatinase, or chitinase. No species is able to utilize
$\mathrm{H}_{2}$ as the sole source of energy and $\mathrm{CO}_{2}$ as the sole source of carbon. All species lack an arginine dihydrolase system. Found in marine habitats. The $\mathrm{G}+\mathrm{C}$ contents of the DNAs range from 52 to $68 \mathrm{~mol} \%$, as determined by buoyant density. The type species is Deleya aesta.

Further descriptive information. Light and electron micrographs showing the general cell morphology and flagellation of representative strains of species of Deleya have been published previously $(4,6)$. There may be difficulty in determining the type of flagellar arrangement in Deleya marina since this species has multitrichous flagella which tend to bend backward toward the cell, giving arrangements which may be interpreted as lateral or peritrichous flagellation. References 4 and 6 should be consulted for a discussion of this problem.

The appearance of the colonies of species of Deleya is not distinctive. All are cream colored and convex with entire edges.

Species of Deleya are able to utilize 47 to 85 different organic compounds as sole sources of carbon and energy. These include pentoses, hexoses, disaccharides, sugar acids, sugar alcohols, fatty acids, dicarboxylic acids, tricarboxylic acid cycle intermediates, amines, and a number of simple aromatic compounds (see above; Table 1). Aromatic compounds are utilized via the $\beta$-ketoadipate pathway, which involves the $o$ cleavage of diphenolic compounds $(4,21)$. DGlucose and D-fructose are utilized via the Entner-Doudoroff pathway $(1,17)$. In the case of the latter sugar, the initial reactions appear to involve the conversion of $D$-fructose to fructose 1- phosphate via a D-fructose:phosphotransferase system, 1-phosphofructokinase, and a pyrophosphate-dependent 6-phosphofructokinase (17).

Species of the genus Deleya. The physiological and nutritional characteristics of the species, as well as their type strains, are presented above and in Table 1. Selected properties which distinguish among the species are presented in Table 2 . Below are the new species combinations. 
TABLE 2-Continued

\begin{tabular}{ccccccccccc}
\hline $\begin{array}{c}\text { Aconi- } \\
\text { tate }\end{array}$ & $\begin{array}{c}\text { Man- } \\
\text { nitol }\end{array}$ & $\begin{array}{c}\delta \text {-Amino- } \\
\text { valerate }\end{array}$ & $\begin{array}{c}\text { L-Histi- } \\
\text { dine }\end{array}$ & $\begin{array}{c}\text { L-Tyro- } \\
\text { sine }\end{array}$ & $\begin{array}{c}\text { DL-Kyn- } \\
\text { urenine }\end{array}$ & $\begin{array}{c}\text { Ethanol- } \\
\text { amine }\end{array}$ & $\begin{array}{c}\text { Benzyl- } \\
\text { amine }\end{array}$ & $\begin{array}{c}\text { Putres- } \\
\text { cine }\end{array}$ & $\begin{array}{c}\text { Sarco- } \\
\text { sine }\end{array}$ & $\begin{array}{c}\text { Allan- } \\
\text { toin }\end{array}$ \\
\hline- & + & - & - & - & - & - & - & - & - & - \\
+ & + & $(4)$ & $(4)$ & + & - & - & - & + & + & + \\
$(5)$ & - & + & + & + & + & - & $(5)$ & + & + & $(5)$ \\
$(13)$ & $(12)$ & + & $(12)$ & + & - & $(13)$ & - & + & + & + \\
+ & + & - & - & + & - & - & - & - & $(6)$ & - \\
\hline
\end{tabular}

Deleya aesta comb. nov. (basionym, Alcaligenes aestus). Motile by means of peritrichous flagella. Utilizes 47 to 53 organic compounds, including hexoses and disaccharides but not pentoses. Only species of Deleya that is able to utilize suberate. The $\mathrm{G}+\mathrm{C}$ content of the DNA is 57 to $58 \mathrm{~mol} \%$, as determined by buoyant density. The type strain is strain 134 (= ATCC 27128).

Deleya pacifica comb. nov. (basionym, Alcaligenes pacificus). Motile by means of peritrichous flagella. Utilizes 55 to 60 organic compounds, including D-glucose and D-fructose but no other hexose or disaccharide. Only species of Deleya that is able to utilize DL-kynurenine. The $\mathrm{G}+\mathrm{C}$ content of the DNA is 67 to $68 \mathrm{~mol} \%$, as determined by buoyant density. The type strain is strain 62 (= ATCC 27122).

Deleya cupida comb. nov. (basionym, Alcaligenes cupidus). Motile by means of peritrichous flagella. Utilizes 69 to 85 organic compounds, including pentoses, hexoses, and disaccharides. Only species of Deleya that is able to utilize Larabinose, D-mannose, saccharate, and glycolate. The $\mathrm{G}+\mathrm{C}$ content of the DNA is 60 to 63 mol\%, as determined by buoyant density. The type strain is strain 79 (= ATCC 27124).

Deleya venusta comb. nov. (basionym, Alcaligenes venustus). Motile by means of peritrichous flagella. Utilizes 52 to 76 organic compounds, including D-glucose, D-fructose, and disaccharides but no additional hexoses or any pentoses. Able to grow at $4^{\circ} \mathrm{C}$. The $\mathrm{G}+\mathrm{C}$ content of the DNA is 52 to $55 \mathrm{~mol} \%$. The type strain is strain 86 (= ATCC 27125).

Deleya marina comb. nov. (basionym, Pseudomonas marina). Motile by means of two to five flagella which are present at one pole of the cell. Utilizes 35 to 47 organic compounds, including D-ribose, D-glucose, D-galactose, and Dfructose but no other pentose, hexose, or disaccharide. Able to grow at $4^{\circ} \mathrm{C}$. The $\mathrm{G}+\mathrm{C}$ content of the DNA is 62 to $64 \mathrm{~mol} \%$. The type strain is strain 219 (= ATCC 25374).

Differentiation from phenotypically similar genera. Species of Deleya are found in marine environments and, therefore, need to be distinguished from the described marine species of
Pseudomonas and Alteromonas (4, 6; P. Baumann, M. Gauthier, and L. Baumann, in Bergey's Manual of Systematic Bacteriology, 9th ed., in press). The presence of peritrichous flagella differentiates most of the species of Deleya from these polarly flagellated genera. All species of Deleya, including the polarly flagellated organism $D$. marina, differ from the two described marine species of Pseudomonas, Pseudomonas doudoroffi and Pseudomonas nautica, by their ability to utilize D-glucose, Dgluconate, and glycerol, properties absent in the latter two species. All species of Deleya accumulate poly- $\beta$-hydroxybutyrate, a trait absent in Alteromonas. Species of Deleya do not produce an extracellular gelatinase or lipase; this distinguishes them from all Alteromonas species except Alteromonas communis and Alteromonas vaga, two species which are sufficiently different from the other Alteromonas species to warrant removal from this genus (12). Species of Deleya can be readily distinguished from terrestrial Pseudomonas and Alcaligenes species by their requirement for at least $75 \mathrm{mM} \mathrm{NaCl}$ for optimal growth rate and yield.

\section{ACKNOWLEDGMENTS}

We thank J. G. Holt for help with nomenclature.

This study was supported by grant DEB-8205938 from the National Science Foundation, as well as by the Aquaculture Program, University of California Agriculture Experiment Station.

\section{LITERATURE CITED}

1. Baumann, L., and P. Baumann. 1973. Enzymes of glucose catabolism in cell-free extracts of non-fermentative marine eubacteria. Can J. Microbiol. 19:302-304.

2. Baumann, L., and P. Baumann. 1974. Regulation of aspartokinase activity in non-fermentative, marine eubacteria. Arch. Microbiol. 95:1-18.

3. Baumann, L., and P. Baumann. 1978. Studies of relationship among terrestrial Pseudomonas, Alcaligenes, and enterobacteria by an immunological comparison of glutamine synthetase. Arch. Microbiol. 119:25-30.

4. Baumann, L., P. Baumann, M. Mandel, and R. D. Allen. 1972. Taxonomy of aerobic marine eubacteria. J. Bacteriol. 110:402-429.

5. Baumann, P., and L. Baumann. 1975. Catabolism of Dfructose and D-ribose by Pseudomonas doudoroffii. I. Physiological studies and mutant analysis. Arch. Microbiol. 105:225-240.

6. Baumann, P., and L. Baumann. 1981. The marine gramnegative eubacteria: genera Photobacterium, Beneckea, 
Alteromonas, Pseudomonas, and Alcaligenes, p. 13021331. In M. P. Starr, H. Stolp, H. G. Trüper, A. Ballows, and H. G. Schlegel (ed.), The prokaryotes. SpringerVerlag, Berlin.

7. Byng, G. S., R. J. Whitaker, R. L. Gherna, and R. A. Jensen. 1980. Variable enzymological patterning in tyrosine biosynthesis as a means of determining natural relatedness among Pseudomonadaceae. J. Bacteriol. 144:247257.

8. Cohen, G. N., R. Y. Stanier, and G. Le Bras. 1969. Regulation of the biosynthesis of amino acids of the aspartate family in coliform bacteria and pseudomonads. J. Bacteriol. 99:791-801.

9. Davis, D. H., R. Y. Stanier, M. Doudoroff, and M. Mandel. 1970. Taxonomic studies of some gram negative, polarly flagellated "hydrogen bacteria" and related species. Arch. Mikrobiol. 70:1-13.

10. De Ley, J. 1978. Modern molecular methods in bacterial taxonomy: evaluation, application, prospects, p. 347-357. In Proceedings of the Fourth International Conference on Plant Pathology and Bacteriology. Angers, vol. 1. GibertClarey, Tours

11. De Ley, J., P. Segers, and M. Gillis. 1978. Intra- and intergeneric similarities of Chromobacterium and Janthiobacterium ribosomal ribonucleic acid cistrons. Int. J. Syst. Bacteriol. 28:154-168.

12. De Smedt, J., M. Bauwens, R. Tytgat, and J. De Ley. 1980. Intra- and intergeneric similarities of ribosomal ribonucleic acid cistrons of free-living, nitrogen-fixing bacteria. Int J. Syst. Bacteriol. 30:106-122.

13. De Smedt, J., and J. De Ley. 1977. Intra- and intergeneric similarities of Agrobacterium ribosomal ribonucleic acid cistrons. Int. J. Syst. Bacteriol. 27:222-240.

14. Fox, G. E., E. Stackebrandt, R. B. Hespell, J. Gibson, J. Maniloff, T. A. Dyer, R. S. Wolfe, W. E. Balch, R. S. Tanner, L. J. Magrum, L. B. Zablen, R.Blakemore, R. Gupta, L. Bonen, B. J. Lewis, D. A. Stahl, K. R. Luehrsen, K. N. Chen, and C. R. Woese. 1980. The phylogeny of prokaryotes. Science 209:457-463.

15. Kersters, K., and J. De Ley. 1980. Classification and identification of bacteria by electrophoresis of their proteins, p. 273-298. In M. Goodfellow, and R. G. Board (ed.), Microbiological classification and identification. Academic Press, Inc., London.

16. Palleroni, N. J., R. Kunisawa, B. Contopoulou, and $M$ Doudoroff. 1973. Nucleic acid homologies in the genus Pseudomonas. Int. J. Syst. Bacteriol. 23:333-339.

17. Sawyer, M. H., P. Baumann, and L. Baumann. 1977 Pathways of D-fructose and D-glucose catabolism in marine species of Alcaligenes, Pseudomonas marina, and Alteromonas communis. Arch. Microbiol. 112:169-172.

18. Sawyer, M. H., P. Baumann, L. Baumann, S. M. Berman, and J. L. Cánovas. 1977. Pathways of D-fructose catabolism in species of Pseudomonas. Arch. Microbiol. 112:49 55 .

19. Stackebrandt, E., and C. R. Woese. 1981. The evolution of prokaryotes, p. 1-32. In M. J. Carlile, J. F. Collins, and B. E. B. Moseley (ed.), Molecular and cellular aspects of microbial evolution. Cambridge University Press, London.

20. Stanier, R. Y. 1976. Réflexions sur la taxonomie des Pseudomonas. Bull. Inst. Pasteur Paris 74:255-270.

21. Stanier, R. Y., N. J. Palleroni, and M. Doudoroff. 1966 The aerobic pseudomonads: a taxonomic study. J. Gen. Microbiol. 43:159-271.

22. Swings, J., P. De Vos, M. Van den Mooter, and J. De Ley 1983. Transfer of Pseudomonas maltophilia Hugh 1981 to the genus Xanthomonas as Xanthomonas maltophilia (Hugh 1981) comb, nov. Int. J. Syst. Bacteriol. 33:409413.

23. Whitaker, R. J., G. S. Byng, R. L. Gherna, and R. A Jensen. 1981. Comparative allostery of 3-deoxy-D-arabino-heptulosonate 7-phosphate synthetase as an indicator of taxonomic relatedness in pseudomonad genera. J. Bacteriol. 145:752-759. 